\title{
CONTROL OF OPEN QUANTUM SYSTEMS
}

\author{
Y. JAPHA, A.G. KOFMAN AND G. KURIZKI \\ Department of Chemical Physics, The Weizmann Institute of Science \\ Rehovot, 76100 , Israel
}

\begin{abstract}
Spontaneous decay of excited cold atoms into a cavity can drastically affect their translational dynamics, namely, atomic reflection, transmission and localization at the interface. We show that the quantum Zeno effect on excitation decay of an atom is observable in open cavities and waveguides, using a sequence of evolution-interrupting pulses on a nanosecond scale.
\end{abstract}

PACS numbers: $42.50 . \mathrm{Lc}, 03.65 . \mathrm{Bz}, 03.75 . \mathrm{Be}, 42.50 .-\mathrm{p}$

\section{Transmission of emitting tunneling atoms in cavities}

Spontaneous emission in atomic tunneling has been virtually unexplored before our recent work [1]. Since tunneling is a distinct manifestation of wave-like properties, it is important to raise the basic questions: can spontaneous decay of internal excitations in tunneling atoms be viewed as a decoherence process that is analogous to its counterpart in diffracted atoms? and if so, how would such decoherence manifest itself?

We have put forward a theory of spontaneous emission from a two-level atom as it tunnels through a square potential barrier [1]. Our theory demonstrates that the emission process is describable as loss of coherence between interfering classical trajectories in space-time, which constitute the atom tunneling motion. The emitted photon at each frequency is correlated to particular atomic classical trajectories, in a way which makes them measurably distinguishable. This distinguishability destroys their interference [2], as does "which-way" ("Welcher-Weg") information, which is obtainable from spontaneous emission in diffracted atoms $[3,4]$.

The ensuing analysis rests on two observations. (i) The overall duration of the decay process is much longer than the inverse transition frequency $\omega_{e g}^{-1}$ (see below). This allows us to resort to the rotating wave approximation (RWA), which is used in the Wigner-Weisskopf (WW) treatment of spontaneous emission [5]. (ii) Nearly all of the cavity-enhanced spontaneous emission is funneled into the continuum of nearly resonant modes with wave-vectors $q \approx(\omega / c) \widehat{z}$, which are aligned with the cavity axis $z$, perpendicular to the atomic incidence axis $x$. This allows us to use the dipole approximation, since $\boldsymbol{q} \cdot \boldsymbol{x} \approx 0$, and neglect off-axis photon recoil effects on the atomic wave packet. Hence, the RWA interaction Hamiltonian of the atom with the cavity-mode continuum becomes effectively one-dimensional, 
$H_{\text {int }}=-\zeta(x) \int \mathrm{d} \omega \rho(\omega)\left[g_{\omega} a_{\omega}|e\rangle\langle g|+\right.$ h.c. $]$. Here $\zeta(x)=1$ for $0 \leq x \leq L$ and 0 elsewhere, i.e., the interaction is confined to the cavity, whose $x$-axis extent coincides with that of the barrier; $\rho(\omega)$ is a Lorentzian mode-density distribution associated with the cavity-mode line width $\eta[6] ; g_{\omega}$ is the coupling of the atom to the cavity mode at $\omega$ and $a_{\omega}$ is the corresponding annihilation operator. The transition frequency $\omega_{e g}$ is shifted (renormalized) by the difference between the AC Stark shifts of $|e\rangle$ and $\left.|g\rangle, \Delta_{\mathrm{AC}}=\vdots \Omega_{e}^{2} / \delta_{e}-\Omega_{g}^{2} / \delta_{g}\right)$.

In order to analyze the entanglement of emitted photon states with the translational degrees of freedom of the tunneling atom, we have developed a theoretical approach which combines the WW treatment [5], resulting in exponential decay of the excited state, with the Feynman path-integral method, which yields a coherent sum over the atomic classical trajectories contributing to tunneling [7].

The above analysis yields the probability for an atom incident as a nearly monochromatic wave packet to be transmitted in the excited state

$$
P_{e}^{\mathrm{tr}}=\left|\sigma\left(E_{k}, V-\mathrm{i} \hbar \Gamma\right)\right|^{2},
$$

where $\sigma\left(E_{k}, V\right)$ is the transnission amplitude for a structureless particle of kinetic energy $E_{k}$ through a square potential barrier of height $V$ and length $L$,

$$
\sigma\left(E_{k}, V\right)=\left[\cos (p L)-\mathrm{i} \frac{k^{2}+p^{2}}{2 k p} \sin (p L)\right]^{-1}
$$

$k=\sqrt{2 m E_{k}} / \hbar$ and $p=\sqrt{2 m\left(E_{k}-V\right)} / \hbar$ being the corresponding wave vectors outside and inside the barrier, respectively. The effect of spontaneous emission is to shift the effective potential $V$ by $-\mathrm{i} \hbar \Gamma$.

Plots of Eq. (1) reveal the overall diminishing of $P_{e}^{\text {tr }}$ with $\gamma$ in both the tunneling (below-barrier) and allowed (above-barrier) regimes of $E_{k}$. The corresponding probability $P_{g}^{\operatorname{tr}}$ of the transmitted ground state wave-packet is an incoherent sum (integral) of partial wave-packet transmission probabilities $P_{\omega}$ associated with photon emission at $\omega$

$$
\begin{aligned}
& P_{g}^{\mathrm{tr}}=\int_{0}^{E_{k}} \mathrm{~d} \omega P_{\omega}, \\
& P_{\omega}=\mathcal{F}(\omega) \sqrt{1-\frac{\hbar \Delta}{E_{k}}}\left|\sigma_{\omega}\left(E_{k}, V\right)\right|^{2},
\end{aligned}
$$

where $\mathcal{F}(\omega)=\rho(\omega)\left|g_{\omega}\right|^{2} /\left(\mathcal{A}^{2}+\gamma^{2}\right)$ and $\sigma_{\omega}\left(E_{k}, V\right)$ is a complicated function of $E_{k}, V$ and $\omega$. The most salient effect of spontaneous emission is seen to be (Fig. 1a) the huge enhancement of $P_{g}^{\mathrm{tr}}$ as a function of $\gamma$ for atoms initially in the deep tunneling regime $p L=\sqrt{2 m\left(V-E_{k}\right)} L / \hbar>1$.

In order to gain more insight into the above general results, we shall henceforth assume that the cavity line width $\eta$ and $E_{k}$ satisfy the following inequalities:

$$
\left|E_{k}-V\right| \ll \hbar \eta<E_{k}<\hbar \omega_{e g}, \gamma \ll \eta \text {. }
$$

The spectrum of spontaneous emission is then limited to $|\Delta| \ll E_{k}$ and becomes Lorentzian in this range, $\mathcal{F}(\omega) \approx \mathcal{L}_{\gamma}(\Delta)$, since the spectral variation of $\rho(\omega)$ and $\left|g_{\omega}\right|^{2}$ is slow, $\rho(\omega)\left|g_{\omega}\right|^{2} \approx 2 \pi \gamma$, in accordance with the WW approximation. The equation for $\sigma_{\omega}$ can now be simplified to

$$
\sigma_{\omega}\left(E_{k}, V\right) \approx \sigma\left(E_{k}-\hbar \Delta, V\right)-\sigma\left(E_{k}, V-\mathrm{i} \hbar \Gamma\right) .
$$


(a)

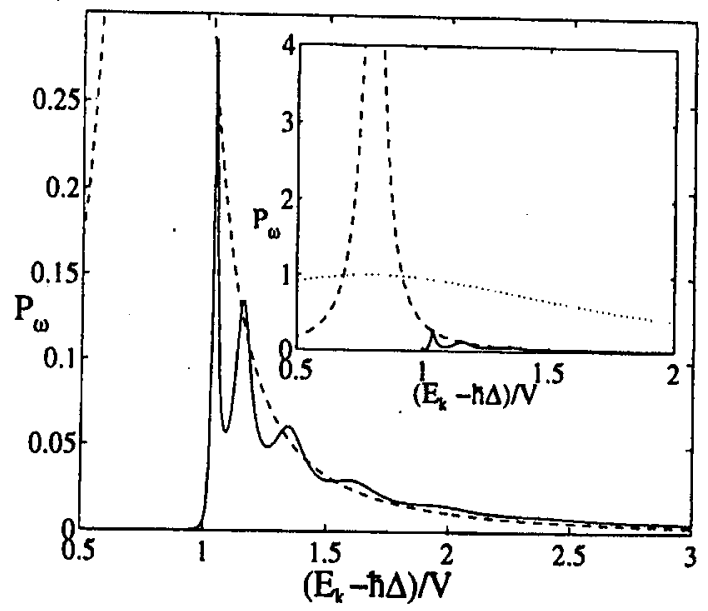

(b)

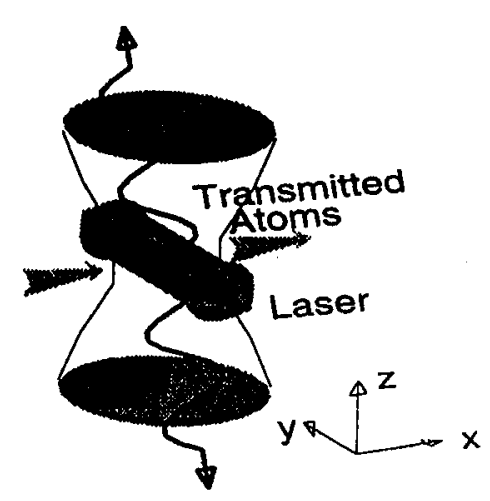

Fig. 1. (a) The energy spectrum of transmitted ground state atoms. Solid curve - transmission probability $P_{\omega}$ [Eq. (3)] (in units of $\hbar / V$ ) for $E_{k} / V=0.8, L=$ $2.5 \lambda_{\mathrm{DB}}\left(E_{k} / V=1\right), \gamma=0.05 \mathrm{~V} / \hbar, \omega_{e g}=100 \mathrm{~V} / \hbar$ as a function of kinetic energy following emission. Dashed curve - spontaneous line shape. Inset: idem, on a small scale. Dotted curve - cavity line shape. (b) Schematic description of the experiment.

It is seen from Eqs. (3) and (5) that the dramatic enhancement effects in the tunneling regime are due to the first term in (5), corresponding to atoms that have decayed to the ground state shortly after entering the barrier and are subsequently transmitted through the barrier as unexcited atoms with kinetic energy $E_{k}-\hbar \Delta$, which can be above the barrier if $\Delta<0$. By contrast, the second term in (5) corresponds to atoms that have decayed shortly before exiting the barrier after having effectively been transmitted as excited atoms with the initial kinetic energy $E_{k}$, whence this term is exponentially small in the tunneling regime. The use of Eq. (5) in Eq. (3) therefore leads to the enhancement of $P_{\omega}$ (Fig. 1a) and $P_{g}^{\operatorname{tr}}$ due to the possibility to gain kinetic energy from the broad vacuum field reservoir by emitting a photon detuned below the resonance $\hbar \omega_{e g}$. In the deep tunneling regime, assuming that $\gamma \ll\left(V-E_{k}\right)$, Eqs. (3)-(5) allow us to roughly estimate that the atoms have probability of order

$$
P_{g}^{\operatorname{tr}} \sim \int_{V}^{E_{k}+\hbar \omega_{e g}} \mathrm{~d} E \mathcal{L}_{\gamma}\left[\left(E-E_{k}\right) / \hbar\right] \approx \frac{\gamma}{V-E_{k}}
$$

to jump over the barrier into the allowed energy regime by emitting a photon with $\Delta<E_{k}-V<0$ (Fig. 1).

Under the assumptions leading to Eq. (5), along with $\hbar \Delta \ll E_{k}$, we can obtain a simplified expression for the total transmission probability

$$
P_{\mathrm{tot}}^{\mathrm{tr}}=P_{g}^{\mathrm{tr}}+P_{e}^{\mathrm{tr}} \approx \int_{0}^{\infty} \mathrm{d} \tau \int_{0}^{\infty} \mathrm{d} \tau^{\prime} e^{-\gamma\left|\tau-\tau^{\prime}\right|} \widehat{\sigma}^{*}(\tau, V) \widehat{\sigma}\left(\tau^{\prime}, V\right)
$$

where $\widehat{\sigma}(t, V)$, the Fourier transform of $\sigma(E, V)$, is the impulse response (to a temporal $\delta$-function) for transmission of a structureless particle. We thus obtain 
the following important result: the total transmission probability $P_{\text {tot }}^{\text {tr }}$ coincides, in the limit of narrow spontaneous line width $\gamma$ [Eq. (4)], with the transmission probability of a partially incoherent wave packet of a structureless particle with coherence time $\gamma^{-1}$ (see Ref. [8]).

The following conclusions can be inferred from the above analysis. (a) The probability distribution of the transmitted atoms is approximately Lorentzian for final kinetic energies $E_{k}-\hbar \Delta$ above the barrier, whereas their counterparts below the barrier only contribute an exponentially small tail to this distribution. (b) The fact that fast atoms emerging from the barrier are almost always unexcited means that the barrier acts as a "filter" that transmits almost only atoms that have already decayed.

These results open a new vista into the transitior: from quantum dynamics to classicality via decoherence by focusing on the $\mathrm{c}$ lects of excitation decay on atomic tunneling. In the limit of negligible decay $\gamma \rightarrow 0$, which is realizable by detuning the cavity off resonance with $\omega_{e g}$, the excited atomic wave packet with $E_{k}<V$ exhibits tunneling, which is a result of interference between many classical trajectories, and is characterized by exponentially low transmission $P_{e}^{\text {tr }}$ [Eq. (1)]. When $\gamma$ is appreciable, the wave packet is dominated by th. rition that has decohered by decay into the field-mode continuum and has thereby lost its tunneling properties: its energy spread becomes classical (statistical), giving rise to a Lorentzian tail into the above-barrier energy range, thereby allowing for enhancement of the transmission [Eqs. (3),(7)]. The effects of this decoherence on barrier traversal times will be discussed elsewhere.

The results predicted here can be experimentally realized by a variety of cold atoms. In accord with Eq. (4), the lifetime of the $|e\rangle \rightarrow|g\rangle$ transition should preferably be long, above $10^{-6} \mathrm{~s}$. A confocal cavity whose finesse is $\approx 10^{5}$ and subtends a solid angle of $\approx 0.1$ steradians can enhance spontaneous emission rate $\gamma$ by a factor of $\approx 30$. The cavity line width $\eta$ should be much larger than $\gamma$, i.e., preferably above $10 \mathrm{MHz}$. Correspondingly, the potential energy $V$ and the kinetic energy $E_{k}$ must be above $0.1 \mathrm{GHz}$, which requires the laser Rabi frequency $\Omega_{e(g)}$ and detuning $\delta_{e(g)}$ to be well within the GHz range. This implies that the transition frequency $\omega_{e g}$ can lie anywhere between the GHz and the optical ranges.

\section{Atomic reflection and localization at cavity interfaces}

We have recently considered an excited atomic wave packet or an atomic beam propagating from a region where spontaneous emission is negligible $(x<0)$ to a region where spontaneous emission is strongly enhanced $(x>0)$, due to the high density of the electromagnetic field modes. The wave function of the total system (atom plus field) can be written in the following general form in the rotating wave approximation:

$$
|\psi(r, t)\rangle=\widetilde{\psi}_{e}(r, t)|e,\{0\}\rangle+\sum_{\boldsymbol{q}} \tilde{\psi}_{\boldsymbol{q}}(r, t)|g,\{\boldsymbol{q}\}\rangle
$$

where the ket-vector $|e,\{0\}\rangle$ denotes the atom in the excited state with no photons in the field, whereas $|g,\{q\}\rangle$ corresponds to the ground state of the atom with a photon emitted at a mode $\boldsymbol{q}$, and $\widetilde{\psi}_{\boldsymbol{e}(\boldsymbol{q})}$ are the corresponding amplitudes. 
One obtains coupled Schrödinger equations for the envelopes of these states given an atom with initial energy $E$ and transition frequency $\omega_{0}, \psi_{e}(\boldsymbol{r})$ and $\psi_{q}(\boldsymbol{r})$ by assuming $\bar{\psi}_{e, q}(r, t)=\psi_{e, q}(r) \mathrm{e}^{-\mathrm{i}\left(E+\hbar \omega_{0} / 2\right) t}$. Far from the interaction region the solution describes propagation of the atomic wave packet. The total energy of the incident excited atom $E+\hbar \omega_{0}$ is then equal to the kinetic energy of the ground state atom plus the emitted photon energy $\hbar \omega_{q}$.

The coupled equations for $\psi_{e}$ and $\psi q$ yield a complicated integro-differential wave equation for $\psi_{e}\left(r_{e}\right)$, with $\Gamma\left(r, r^{\prime}\right)$ acting as a non-local complex potential whose shape and strength are determined by the confined mode eigenfunctions $\mathcal{E}_{q}(r)$. If the line width of the spatially confined modes $\hbar \eta_{\mathrm{c}}$ is much larger than the atomic energy $E$, the recoil energy $E_{\text {rec }} \equiv \hbar^{2} \omega_{0}^{2} / 2 m c^{2}$ and the spontaneous line width in the confined reservoir, $\hbar \gamma_{c}$, then the correlativis iength of the interaction of the emitted photon with the atom is much shorter than the spontaneous decay length and the de Broglie wavelength $\lambda_{\mathrm{DB}}$. Such an atom effectively moves in a local complex potential

$$
\Gamma(r)=\int \mathrm{d}^{3} \boldsymbol{r}^{\prime} \Gamma\left(\boldsymbol{r}, \boldsymbol{r}^{\prime}\right)=|\mu|^{2} \frac{\sum_{\boldsymbol{q}}\left|\mathcal{E}_{\boldsymbol{q}}(\boldsymbol{r})\right|^{2} \delta\left(\omega_{\boldsymbol{q}}-\omega_{0}\right)}{\Delta_{\mathrm{c}}-\mathrm{i} \eta_{\mathrm{c}}},
$$

where $\mu$ is the atomic dipole matrix element, $\mathcal{E}_{\boldsymbol{q}}(r)$ are the field mode amplitudes and $\Delta_{c}$ is the detuning of the atomic transition frequency $\omega_{0}$ from the center of the spectral line of the reservoir.

In order to concentrate on the atomic motion along the axis of incidence $x$ and avoid diffraction effects caused by the local potential in the directions perpendicular to $x$, we consider a multimode confocal cavity where the many degenerate modes contributing to $\Gamma(r)$ render it approximately uniform in the directions perpendicular to $x$. We assume that the transition frequency $\omega_{0}$ is resonant with the Lorentzian center of the degenerate modes. Then the real part of $\Gamma(x)$ is much less than the imaginary part $\gamma_{c}(x)=\operatorname{Im}\{\Gamma(x)\}$. We then obtain

$$
\frac{\partial^{2}}{\partial x^{2}} \psi_{e}(x)+\frac{2 m}{\hbar^{2}}\left[E+\mathrm{i} \hbar \gamma_{\mathrm{c}}(x)\right] \psi_{e}(x)=0 \text {. }
$$

For a step-like interaction profile $\gamma_{c}(x)=\gamma_{c} \Theta(x)$, where $\Theta(x)$ is the Heaviside step function, the probability to detect an excited atom decreases as $\mathrm{e}^{\mathrm{i} k_{\gamma} x}$, where $k_{\gamma}=\sqrt{2 m\left(E+i \hbar \gamma_{c}\right)} / \hbar$, so that only the fraction $|r|^{2}$ of excited atoms remains at large negative $x$ (to the left of the interface). This reflection increases with the spontaneous emission rate $\gamma_{c}$. The atomic interaction with the confined vacuum reservoir for $\hbar \gamma>E$ is thus analogous to the skin effect of light reflection from metals. If the energy of the incident atom is comparable to $E_{\mathrm{rec}}$, the width $\Delta x$ of the interface should satisfy $\Delta x \approx \lambda_{\mathrm{DB}}(E) \sim \lambda_{\text {opt }}$. A realistic description of the atomic entry into a confocal cavity shows a much lower reflection probability, even for subrecoil energies. However, when the real part of $\Gamma(x)$ contributes too, for $\omega_{0}$ well off the center of the Lorentzian spectrum (large $\Delta_{\mathrm{c}}$ ), the cavity can be strongly reflective. This spectral dependence of the reflectivity on the detuning is characteristic of the atomic skin effect.

The spatial variation of the $q$-mode amplitude in Eq. (8) can be estimated for a strong decay $\hbar \gamma_{\mathrm{c}} \gg E$ and incidence energy well above the recoil limit. Then 
$\psi_{\boldsymbol{q}} \propto \exp \left(+\mathrm{i} k_{\boldsymbol{q}} x\right)$, where $\hbar k_{\boldsymbol{q}}=\sqrt{2 m\left(E-\hbar \Delta_{\boldsymbol{q}}\right)}$ and $\Delta_{\boldsymbol{q}}=\omega_{\boldsymbol{q}}-\omega_{0}$. Whenever $E>\hbar \Delta_{\boldsymbol{q}}, k_{\boldsymbol{q}}$ becomes imaginary and $\psi_{\boldsymbol{q}}(r)$ is exponentially localized at the interface between free space and the confined-field region. A solution with imaginary $k_{q}^{x}$ represents a transient atomic wave packet which disappears after the incident atomic wave packet decays or leaves the interface, and is accompanied by a transient bound photon, which eventually disappears with it, after the time $\sim \hbar / \Delta E$, the inverse of the energy bandwidth $\Delta E$ of the incident atom. If such a photon is detected, then a localized atomic state is formed. The subsequent evolution of the atomic wave packet is governed by the free-space Schrödinger equations with the localized atomic distribution serving as the initial condition.

To conclude, we have found that excited-atom reflection from the interface between two spatial regions with different spontaneous emission rates is appreciable for cold atoms and enhanced coupling to the mode continuum, when the effective width of the interface is smaller than the atomic de Broglie wavelength. This reflection is analogous to the optical skin effect of metal surfaces. Transient localized atomic state appear at the interface while an excited two-level atom is crossing it, due to detection of spontaneously emitted "bound photons" at "forbidden" energies, having short lifetime and range of propagation. The regime considered here is essentially different from Ref. [9], where the correlation time of the atom with the emitted photon is large, thereby responsible for the oscillation of the atomic population.

\section{Quantum Zeno effect on atomic excitation decay in resonators}

The "watchdog" or quantum Zeno effect (QZE) is a spectacular manifestation of the influence of continuous measurements on the evolution of a quantum system. The original QZE prediction has been the inhibition of exponential decay of an excited state into a reservoir, by repeated interruption of the system-reservoir coupling by measurements [10-12].

We have recently demonstrated [13] that the inhibition of nearly-exponential excited-state decay by the QZE in two-level atoms, in the spirit of the original suggestion [10], is amenable to experimental verification in resonators. Although this task is widely believed to be very difficult, we have shown, by means of our unified theory of spontaneous emission into reservoirs with arbitrary mode-density spectra [11], that several realizable configurations based on two-level emitters in cavities or in waveguides are in fact adequate for QZE observation. The possibilities for such observation have been examined in various regimes that can arise in resonators.

We start with a general analysis of the evolution of an initially excited two-level atom coupled to an arbitrary density-of-modes (DOM) spectrum $\rho(\omega)$ of the electromagnetic field in the vacuum state. At time $\tau$ this evolution is interrupted by a short optical pulse, which serves as a quantum measuring device [14-17]. Its role is to break the evolution coherence, by transferring the populations of the excited state $|e\rangle$ to an auxiliary state which then decays back to $|e\rangle$ incoherently [15].

As in our previous treatment [18], the atomic response, i.e., the emission rate into this reservoir at frequency $\omega$, which is $|g(\omega)|^{2} \rho(\omega), \hbar g(\omega)$ being the field-atom 
coupling energy, is divided into two parts,

$$
G(\omega)=G_{\mathrm{s}}(\omega)+G_{\mathrm{b}}(\omega) .
$$

Here $G_{s}(\omega)$ stands for the sharply-varying (nearly-singular) part of the DOM distribution, associated with narrow cavity-mode lines, the frequency cut-off in waveguides, or photonic band edges. The complementary part $G_{\mathrm{b}}(\omega)$ stands for the broad portion of the DOM distribution (the "background" modes), which always coincides with the free-space DOM $\rho(\omega) \sim \omega^{2}$ at frequencies well above the sharp spectral features. In an open structure (see below), $G_{\mathrm{b}}(\omega)$ represents the atom coupling to the unconfined free-space modes.

We cast the excited-state amplitude in the form $\alpha_{e}(\tau) \mathrm{e}^{-\mathrm{i} \omega_{\mathrm{a}} \tau}$, where $\omega_{\mathrm{a}}$ is the atomic resonance frequency. Then, for arbitrary DOM spectra and coupling strengths, one can reduce the equations for spontaneous decay [19] to the following evolution equation, up to the interruption time $\tau$ :

$$
\dot{\alpha}_{e}(\tau)=-\int_{0}^{\tau} \mathrm{d} t\left[\Phi_{\mathrm{s}}(t)+\Phi_{\mathrm{b}}(t)\right] \mathrm{e}^{\mathrm{i} \Delta t} \alpha_{e}(\tau-t) .
$$

Here $\Delta=\omega_{\mathrm{a}}-\omega_{\mathrm{s}}, \omega_{\mathrm{s}}$ is a characteristic frequency corresponding to the maximum or the singularity of the sharp spectral feature, whereas $\Phi_{\mathrm{s}}(t)$ and $\Phi_{\mathrm{b}}(t)$ are the time-domain Fourier-transforms of $G_{\mathrm{s}}(\omega)$ and $G_{\mathrm{b}}(\omega)$, respectively,

$$
\Phi_{\mathrm{s}(\mathrm{b})}(t)=\int_{0}^{\infty} \mathrm{d} \omega G_{\mathrm{s}(\mathrm{b})}(\omega) \mathrm{e}^{-\mathrm{i}\left(\omega-\omega_{\mathrm{s}}\right) t}
$$

Restricting ourselves to sufficiently short interruption intervals $\tau$ such that $\alpha_{e}(\tau) \approx 1$, yet long enough to allow the rotating wave approximation (RWA), Eqs. (10),(11) yield

$$
\alpha_{e}(\tau) \approx 1-\int_{0}^{\tau} \mathrm{d} t(\tau-t) \Phi_{\mathrm{s}}(t) \mathrm{e}^{\mathrm{i} \Delta t}-\gamma_{\mathrm{b}} \tau / 2 .
$$

The terms within the parentheses in Eq. (12) are the contribution of the background DOM, simplified according to the Weisskopf-Wigner approximation [19]. Here $\gamma_{\mathrm{b}}=2 \pi G_{\mathrm{b}}\left(\omega_{\mathrm{a}}\right)$ is the effective rate of spontaneous emission into the background modes.

Equation (12) is obtained to first order in the atom-field interaction. To the same accuracy, the excited state probability after $n$ interruptions (measurements), $W(t=n \tau)=\left|\alpha_{e}(\tau)\right|^{2 n}$, can be written as

$$
W(t=n \tau) \approx\left[2 \operatorname{Re} \alpha_{e}(\tau)-1\right]^{n} \approx \mathrm{e}^{-\kappa t},
$$

where

$$
\kappa=2 \operatorname{Re}\left[1-\alpha_{e}(\tau)\right] / \tau .
$$

In most structures $\gamma_{\mathrm{b}}$ is comparable to $\gamma_{f}$ and gives rise to an exponential decay factor in the excited state probability regardless of how short $\tau$ is, i.e., $\kappa=\kappa_{\mathrm{s}}+\gamma_{\mathrm{b}}$, where $\kappa_{\mathrm{s}}$ is the contribution to $\kappa$ from the sharply-varying modes.

Thus the background-DOM effect cannot be modified by QZE. Only the sharply-varying DOM portion allows for QZE, provided that

$$
\kappa_{\mathrm{s}}=(2 / \tau) \operatorname{Re} \int_{0}^{\tau} \mathrm{d} t(\tau-t) \Phi_{\mathrm{s}}(t) \mathrm{e}^{\mathrm{i} \Delta t}
$$


rises with $\tau$ for sufficiently short $\tau$. This is essentially a condition on the correlation (or memory) time of the field reservoir.

First and foremost, we wish to apply the above analysis to the case of a two-level atom coupled to a near-resonant Lorentzian line centered at $\omega_{s}$, characterizing a high- $Q$ cavity mode or a "defect" mode in a photonic band structure [19]. In this case, $G_{\mathrm{s}}(\omega)=g_{\mathrm{s}}^{2} \Gamma_{\mathrm{s}} /\left\{\pi\left[\Gamma_{\mathrm{s}}^{2}+\left(\omega-\omega_{\mathrm{s}}\right)^{2}\right]\right\}$, where $g_{\mathrm{s}}$ is the resonant coupling strength and $\Gamma_{\mathrm{s}}$ is the line width (Fig. 2 - inset). In the short-time approximation, taking into account that the Fourier transform of the Lorentzian $G_{\mathrm{s}}(\omega)$ is $\Phi_{\mathrm{s}}(t)=g_{\mathrm{s}}^{2} \mathrm{e}^{-\Gamma_{\mathrm{s}} t}$, Eq. (12) yields (neglecting the background modes)

$$
\alpha_{e}(\tau) \approx 1-\frac{g_{\mathrm{s}}{ }^{2}}{\Gamma_{\mathrm{s}}-\mathrm{i} \Delta}\left[\tau+\frac{\mathrm{e}^{\left(\mathrm{i} \Delta-\Gamma_{\mathrm{s}}\right) \tau}-1}{\Gamma_{\mathrm{s}}-\mathrm{i} \Delta}\right] .
$$

The QZE condition is $\tau \ll\left(\Gamma_{\mathrm{s}}+|\Delta|\right)^{-1}, g_{\mathrm{s}}^{-1}$ : obviously, it is easiest to satisfy this inequality on resonance, when $\Delta=0$. Then Eq. (16) yields

$$
\kappa=\kappa_{\mathrm{s}}+\gamma_{\mathrm{b}}, \quad \kappa_{\mathrm{s}}=g_{\mathrm{s}}^{2} \tau .
$$

Only the $\kappa_{\mathrm{s}}$ term decreases with $\tau$, indicating the QZE inhibition of the smooth nearly-exponential decay into the field reservoir as $\tau \rightarrow 0$. Since $\Gamma_{\mathrm{s}}$ and $\Delta$ have dropped out of Eq. (17), the decay inhibition is the same for both strong- and weak-coupling regimes (Fig. 2). Physically, this comes about since for $\tau \ll g_{\mathrm{s}}^{-1}$ the energy uncertainty of the emitted photon is too large to distinguish between reversible and irreversible evolutions.

The experimental scheme we envisage for observing the above effects is as follows. A fraction of an atomic beam oriented perpendicular to the axis of a confocal cavity is excited to state $|e\rangle$ by a laser outside the cavity. Within the cavity the atoms repeatedly interact with a pump laser, which is resonant with the $|e\rangle \rightarrow|u\rangle$ transition frequency. The resulting $|e\rangle \rightarrow|g\rangle$ fluorescence rate is collected as in

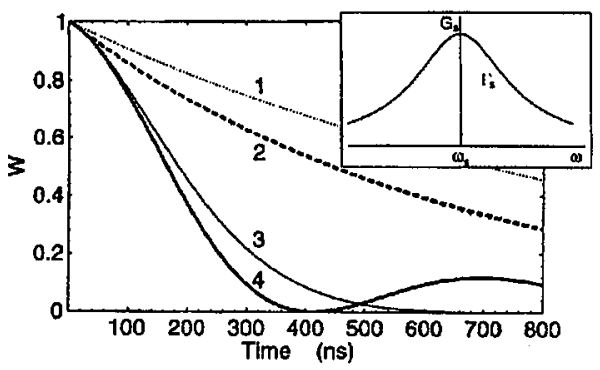

Fig. 2. Evolution of excited-state population $W$ in two-level atom coupled to cavity mode with Lorentzian line shape (inset) in case (i) (on resonance, $\Delta=0$ ): curve 1 decay to background-mode continuum at rate $\gamma_{\mathrm{b}} \approx \gamma_{f}=10^{6} \mathrm{~s}^{-1}$; curve 3 - uninterrupted decay in cavity with $F \equiv(1-R)^{-2}=10^{5}, L=15 \mathrm{~cm}$, and $f=0.02$; curve 4 - idem, but with $F=10^{6}$ (damped Rabi oscillations); curve 2 - interrupted evolution along both curves 3 and 4 , at intervals $\tau=3 \times 10^{-8} \mathrm{~s}$. 


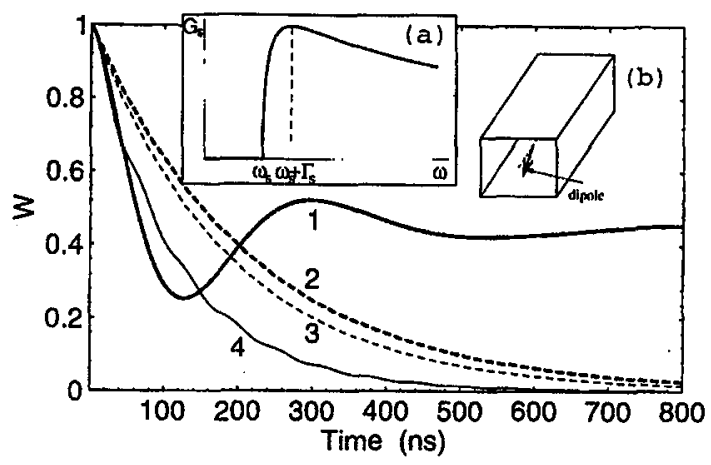

Fig. 3. Idem, for two-level atom $\left(\gamma_{f}=10^{6} \mathrm{~s}^{-1}\right)$ coupled to waveguide field, with coupling $C^{2 / 3}=1.2 \times 10^{7} \mathrm{~s}^{-1}$ and width $\Gamma_{\mathrm{s}}=0$ : curve $1-$ uninterrupted evolution at cut-off frequency $(\Delta=0)$; curve 4 - idem, $\Delta=10^{8} \mathrm{~s}^{-1}$; curve 2 - interrupted evolution at intervals $\tau=10^{-8} \mathrm{~s}$ for $\Delta=0$; curve 3 - idem, for $\Delta=10^{8} \mathrm{~s}^{-1}$. Insets - (a) DOM with cut-off [Eq. (17)]; (b) dipole in a wa veguide.

Ref. [6] and monitored as a function of the pulse repetition rate. Each short, intense pump pulse of duration $t_{\mathrm{p}}$ and Rabi frequency $\Omega_{\mathrm{p}}$ is followed by spontaneous decay (via fluorescence) from $|u\rangle$ back to $|e\rangle$, at a rate $\gamma_{u}$. The "measuring" pulse has to satisfy $t_{\mathrm{p}}{ }^{-1}, \ll \gamma_{u} \ll \Omega_{\mathrm{p}}$, so as to destroy the coherence of the system evolution, on the one hand, and reshuffle the entire population from $|e\rangle$ to $|u\rangle$ and back, on the other hand (Fig. 3 - inset). By combining these requirements with the demand that the interval between measurements significantly exceed the measurement time, we infer the inequality $\tau \gg t_{\mathrm{p}}$. The above inequality can be relaxed to require $\tau \gg \gamma_{u}^{-1}$ if the "measurements" are performed with $\pi$ pulses: $\Omega_{\mathrm{p}} t_{\mathrm{p}}=\pi, t_{\mathrm{p}} \ll \gamma_{u}^{-1}$. The only real constraint is that $\left(\Gamma_{\mathbf{s}}+|\Delta|\right)^{-1} \gg \tau \gg \gamma_{u}^{-1}$. This calls for choosing a $|u\rangle \rightarrow|e\rangle$ transition with a much shorter radiation lifetime than that of $|e\rangle \rightarrow|g\rangle$. The curves in Figs. 2 and 3 are calculated for such a choice, and for feasible cavity parameters: $\Gamma_{\mathrm{s}}=(1-R) c / L, g_{\mathrm{s}}=\sqrt{c f \gamma_{f} /(2 L)}, \gamma_{\mathrm{b}}=(1-f) \gamma_{f}$, where $R$ is the geometric-mean reflectivity of the two mirrors, $f$ is the fractional solid angle (normalized to $4 \pi$ ) subtended by the confocal cavity, and $L$ is the cavity length.

- We now extend the above analysis to any DOM distributions characterized by a cut-off frequency, as in a waveguide, a photonic band edge or a phonon reservoir (with Debye cut-off). A specific model for the spectral response of a DOM distribution with a cut-off is represented by [19] (Fig. 3 - inset (a)).

$$
G_{\mathrm{s}}(\omega)=\left[C \sqrt{\omega-\omega_{\mathrm{s}}} /\left(\omega-\omega_{\mathrm{s}}+\Gamma_{\mathrm{s}}\right)\right] \Theta\left(\omega-\omega_{\mathrm{s}}\right),
$$

where $\omega_{\mathrm{s}}$ is the cut-off (or band-edge) frequency, $\Gamma_{\mathbf{s}}$ is the cut-off smoothing parameter, $C$ is the strength of the coupling of the atomic dipole to this reservoir, and $\Theta(\cdot)$ is the Heaviside step function. Upon computing the Fourier transform of Eq. (18), we find from Eqs. (10),(11) that the QZE condition is

$$
\tau \ll \min \left\{\Gamma_{\mathrm{s}}^{-1},|\Delta|^{-1}, C^{-2 / 3}\right\} \text {. }
$$

Under this condition, Eqs. (10) and (15) yield $\alpha_{e}(\tau)$ of the form

$$
\kappa_{\mathrm{s}}=\left(2^{5 / 2} \pi^{1 / 2} / 3\right) C \tau^{1 / 2} \text {. }
$$


As mentioned above, the QZE is now less pronounced (see Fig. 3, where we used the exact solution to compute $\left.\alpha_{e}(\tau)\right)$. This case is realizable for an active dipole layer embedded in a dielectric waveguide, using a level scheme similar to that of Fig. 2.

Instead of disrupting the coherence of the evolution by a sequence of "impulsive" measurements, i.e., short $\pi$-pulses, we can achieve this goal by noisy-field dephasing of $\alpha_{e}(t)$. Random ac-Stark shift by an intensity-fluctuating field results in

$$
\kappa_{\mathrm{s}}=\int G_{\mathrm{s}}\left(\Delta+\omega_{\mathrm{a}}\right) F(\Delta) \mathrm{d} \Delta,
$$

where $F(\Delta)$ is a Lorentzian spectrum whose width is $\left\langle\Delta \omega^{2}\right\rangle \tau_{\mathrm{c}}$, the product of the mean-square Stark shift and the noisy-field correlation time.

Our unified analysis of two-level system coupling to field reservoirs has revealed the general optimal conditions for observing the QZE in various structures (cavities, waveguides, phonon reservoirs, and photonic band structures). We note that the wave function collapse notion is not involved here, since the measurement is explicitly described as an act of coherence-breaking [15]. This analysis also clarifies that QZE cannot combat the background-modes contribution to exponential decay, and is therefore inadequate for decoherence error prevention [20]. The best way to achieve such prevention is by switching-off the entire density of modes, i.e., placing the resonance well within an ideal band gap.

\section{References}

[1] Y. Japha, G. Kurizki, Phys. Rev. Lett. 77, 2909 (1996).

[2] D. Sokolovski, J.N.L. Connor, Phys. Rev. A 47, 4677 (1993) noted the connection between traversal-time measurement in tunneling and path information.

[3] T. Pfau, S. Spälter, Ch. Kurtsiefer, C.R. Ekstrom, J. Mlynek, Phys. Rev. Lett. 73, 1223 (1994); P.L. Gould, P.J. Martin, G.A. Ruff, R.E. Stoner, J.-L. Picque, D.E. Pritchard, Phys. Rev. A 43, 585 (1991).

[4] A. Stern, Y. Aharonov, Y. Imry, Phys. Rev. A 41, 3436 (1990).

[5] C. Cohen-Tannoudji, J. Dupont-Roc, G. Grynberg, Atom-Field Interactions, Wiley, New York 1992; G.S. Agarwal, Quantum Statistical Theories of Spontaneous Emission, Springer, Berlin 1974.

[6] D.J. Heinzen, J.J. Childs, J.E. Thomas, M.S. Feld, Phys. Rev. Lett. 58, 1320 (1987).

[7] R.P. Feynman, A.R. Hibbs, Quantum Mechanics and Path Integrals, McGraw-Hill, New York 1965.

[8] Y. Japha, V.M. Akulin, G. Kurizki, submitted to Phys. Rev. Lett.

[9] B.G. Englert, J. Schwinger, A.O. Barut, M.O. Scully, Europhys. Lett. 14, 25 (1991); M.O. Scully, G.M. Meyer, H. Walther, Phys. Rev. Lett. 76, 4144 (1996).

[10] B. Misra, E.C.G. Sudarshan, J. Math. l'hys. 18, 756 (1977).

[11] J. Maddox, Nature 306, 111 (1983).

[12] A. Peres, Phys. Rev. D 39, 2943 (1989).

[13] A.G. Kofman, G. Kurizki, Phys. Rev. A 54, R3750 (1996).

[14] W.M. Itano, D.J. Heinzen, J.J. Bollinger, D.J. Wineland, Phys. Rev. A 41, 2295 (1990); R. Cook, Phys. Scr. Vol. T 21, 49 (1988). 
[15] P.L. Knight, Nature 344, 493 (1990); T. Petrosky, S. Tasaki, I. Prigogine, Phys. Lett. A 151, 109 (1990); E. Block, P.R. Berman, Phys. Rev. A 44, 1466 (1991); L.E. Ballentine, ibid. 43, 5165 (1991); V. Frerichs, A. Schenzle, ibid. 44, 1962 (1991).

[16] M.B. Plenio, P.L. Knight, R.C. Thompson, Opt. Commun. 123, 278 (1996).

[17] A. Luis, J. Periina, Phys. Rev. Lett. 76, 4340 (1996).

[18] G. Kurizki, A.G. Kofman, V. Yudson, Phys. Rev. A 53, R35 (1996).

[19] A.G. Kofman, G. Kurizki, B. Sherman, J. Mod. Opt. 41, 353 (1994).

[20] L. Vaidman, L. Goldenberg, S. Wiesner, preprint quant-ph/9603031 (1996); S.L. Braunstein, preprint quant-ph/9604036 (1996). 